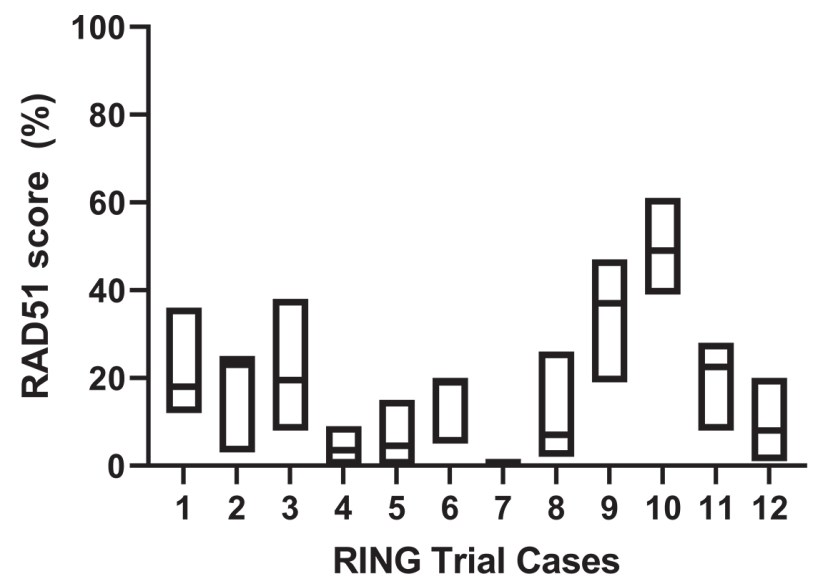

Abstract 376 Figure 1 Violin plot showing the varaiability in RAD51 scores (foci cut-off: $n=3$ ) between four observers. RAD51 scores were based on centrally stained slides. Lines in the plot indicate the median, min and max and RAD51 scores

participating centers for local RAD51 scoring. For the scope of the RING trial, a predefined and uniform scoring methodology was applied. Scoring was performed blinded for genetic and clinical data. Specific features in the analysis of the co-IF, including the number of RAD51 foci per nucleus and the presence of RAD51 subclonality, i.e., distinct RAD51 positive and negative areas, were incorporated in the RAD51 scoring form. For non-normally distributed data, variability was analyzed using the median, $25^{\text {th }}$ percentile $(\mathrm{Q} 1)$ and $75^{\text {th }}$ percentile (Q3).

Result(s)* Median variability in RAD51 scores between observers in centrally stained slides was 21\% (Q1: 15\%; Q3: 24\%) (figure 1). For the majority of cases $(n=10 / 12)$, a limited interobserver variability, defined as $\geq 3$ observers with scores in a narrow range, was detected. In contrast, in cases where observers noted granular pannuclear RAD51 staining or RAD51 background, there was a substantial variability in scores (figure 1; case 6 and 8). Median variability in RAD51 scores between centrally and locally stained IF slides was 7.7\% (Q1: 4.1\%; Q3: 11.7\%).

Conclusion* This is the first cross-European interlaboratory assessment of the performance of RAD51/Geminin co-IF. We show that subtle local protocol differences do not impact final RAD51 scores. Furthermore, we elucidated features that may negatively impact RAD51 score accuracy.

\section{EFFICACY ANALYSIS OF NIRAPARIB USING PATIENT- DERIVED XENOGRAFT OF RARE SUBTYPES OF OVARIAN CANCER}

${ }^{1} \mathrm{H}$ Asano*, ${ }^{2,3} \mathrm{KC}$ Hatanaka, ${ }^{4} \mathrm{Y}$ Matsuno, ${ }^{2,3} \mathrm{Y}$ Hatanaka, ${ }^{1} \mathrm{H}$ Watari. ${ }^{1}$ Hokkaido University, Department of Obstetrics and Gynecology, Sapporo, Japan; ${ }^{2}$ Hokkaido University Hospital, Center for Development of Advanced Diagnostics, Sapporo, Japan; ${ }^{3}$ Hokkaido University Hospital, Research Division of Genome Companion Diagnostics, Sapporo, Japan; ${ }^{4}$ Hokkaido University Hospital, Department of Surgical Pathology, Sapporo, Japan

\subsection{6/ijgc-2021-ESG0.593}

Introduction/Background* Niraparib, a PARP inhibitor, causes synthetic lethality in tumors with homologous recombination deficiency (HRD) and is now approved for ovarian cancer.
However, the genetic alterations related to HRD rarely occur in ovarian cancers other than high-grade serous carcinoma or endometrioid carcinoma, thus the efficacy of niraparib for rare subtypes of ovarian cancer remains unclear. In this study, we investigated the efficacy of niraparib using Patient-DerivedXenograft (PDX) models with rare subtypes of ovarian cancer and correlated between the efficacy and the expression levels of SLFN11 and ARID1A, a sensitizer of DNA-targeted therapies and a key component of the SWI/SNF complex, respectively.

Methodology We consecutively collected a total of 11 tissue specimens with a preoperative diagnosis of ovarian cancer which was surgically resected in our hospital. These tumors were directly transplanted subcutaneously into NOG mice and PDXs were established. The histologic characteristics were compared between parental tumors and PDX ones. Immunohistochemical (IHC) staining for ARID1A (D2A8U, Cell Signaling Technology) and SLFN11(D-2 sc-515071X, Santa Cruz) and genetic alterations of 160 cancer-related genes including ARID1A (Human Comprehensive Cancer Panel, Qiagen) were performed using the parental tumors. Response to carboplatin (CBDCA) and niraparib was analyzed using the PDX models.

Result(s)* PDXs were established for each one case of carcinosarcoma (CS), adenocarcinoma (Adeno) with neuroendocrine carcinoma (NEC), and clear cell carcinoma (CCC). In histological comparison, PDX tumors generally mimicked the parental tumors. Loss of ARID1A in IHC was found in CCC and CS cases, and genetic alteration of ARID1A was also detected in the CCC case. Positive staining for SLFN11 was found in CS and Adeno with NEC, both of which had TP53 alterations. In the PDX of CS, both CBDCA and niraparib suppressed tumor growth in a dose-dependent manner. In the PDX of Adeno with NEC, CBDCA significantly suppressed tumor growth, while niraparib did not. The efficacy of CBDCA and niraparib is currently under consideration in the PDX of CCC.

Conclusion* The combination of ARID1A and SLFN11 in IHC may be an efficacy biomarker for niraparib in rare subtypes of ovarian cancer. We plan to increase the sample size in the future. 